\title{
La femme au colt 45 : un parcours dans l'imaginaire aquatique de Marie Redonnet
}

\author{
González Hernández, Ana Teresa
}

Université de Salamanca, anat@usal.es

\begin{abstract}
Resumen
Bajo la apariencia de una escritura límpida y una narrativa sin artificios, Marie Redonnet (1948) presenta un universo ficcional complejo, con personajes solitarios y desvalidos -femeninos en su mayor parte-, sometidos a grandes tensiones sociales, anclados en un marco espacio-temporal indefinido $y$, por lo general, inmersos en un estado de abandono existencial, de estancamiento emocional al que viene a contribuir la presencia de un componente clave del imaginario redonnetiano : el elemento acuático. En Redonnet, las diferentes manifestaciones del agua : océanos, mares, ríos, lagos, cascadas, pantanos etc, se convierten en verdaderas metáforas recurrentes, incluso obsesivas, de rico significado. Tomando como punto de partida de nuestro estudio la trilogía: Splendid Hôtel, Forever Valley y Rose Mélie Rose, novelas publicadas entre 1986 y 1987, nos proponemos analizar, en un primer momento, el papel que desempeña la presencia recurrente de la temática del agua en dicha trilogía. Fijaremos, después, nuestra atención en el estudio de la última novela de Marie Redonnet : La femme au colt 45, publicada en 2016. En ella, con un estilo depurado y minimalista, cercano al lenguaje dramatúrgico, la autora plantea, a través de la experiencia vital de Lora Sander, una actriz que huye de la dictadora de su país, para iniciar una nueva vida en otro lugar, del "otro lado del río". El análisis de la presencia recurrente del agua en esta obra, sus distintas manifestaciones, así como el significado de los distintos elementos asociados a la misma, nos permitirá trazar la evolución del tratamiento del imaginario acuático en la creación literaria de Redonnet.
\end{abstract}

Palabras clave : escritura minimalista ; imaginario acuático ; río ; isla ; arca.

\section{Résumé}

Sous l'apparence d'une écriture dépouillée et une narration dénudée, Marie Redonnet (1948), nous présente un univers fictionnel complexe, peuplé de personnages démunis et solitaires -féminins pour la plupart-, soumis aux grandes tensions sociales, ancrés dans un cadre spatio-temporel flou, et fréquemment plongés dans un état d'abandon existentiel, de stagnation émotionnelle, auquel vient contribuer la présence d'une composante clé de l'imaginaire redonnetien : l'élément aquatique. Chez Redonnet, les différentes variantes de l'eau: océans, mers, fleuves, lacs, cascades, marécages, etc, deviennent de véritables métaphores récurrentes, voire obsédantes, riches de signification. Prenant comme point de départ de notre parcours la trilogie : Splendid Hôtel, Forever Valley et Rose Mélie Rose, publiée entre 1986 et 1987, nous nous proposons d'analyser, dans un premier temps, le rôle et la signification de la présence récurrente de la thématique de l'eau dans cette trilogie. Nous dresserons, ensuite, le tableau des différentes manifestations de l'eau et des divers aspects de la symbolique aquatique dans son dernier roman : La femme au colt 45, paru en 2016. Dans ce roman, utilisant une langue épurée et minimaliste, proche du langage dramaturgique, Marie Redonnet nous pose la problématique de la précarité des réfugiés, pour recommencer une nouvelle vie ailleurs, de "l'autre côté du fleuve », à travers l'expérience vitale de Lora Sander, une comédienne qui fuit la dictature de son pays. L'étude dans ce dernier roman de la présence de l'eau, sous toutes ses manifestations, ainsi que la signification des différents élements qui lui sont associés, nous permettra de nous interroger sur l'évolution du traitement de l'imaginaire aquatique dans la création littéraire de Marie Redonnet.

Mots-clés : écriture minimaliste ; imaginaire aquatique ; fleuve ; île ; arche. 


\begin{abstract}
Under the look of a clean way of writing and a fireworkless narrative, Marie Redonnet (1948) unveils a fictional and complex universe, inhabited by lonely and damageable characters- most of them femalesubjected to big social stress, in an unknow place or time and, usually put to an existential abandonment. To this adjoins the presence of a key component in the redonnian worldview : water bodies. Since with Redonnet, oceans, seas, rivers, lakes, waterfalls, swamps etc... become real and iterative, even obsessive metaphors full of significance. Taking as starting point the trilogy: Splendid Hôtel, Forever Valley and Rose Mélie Rose (published between 1986 and 1987), in this study we intend to analize, first of all, the importance of the recurrent appearence of water in Redonnet's work. After that, we will focus on her last novel : La femme au colt 45, (published in 2016), where we find a pure and minimalist style, close to the dramatic language. Throughout the life of Lora Sander, an actress who flees of the dictatorship of her country, to begin a new life in another place " across the pond». The study of the repeated reference to different water bodies, along with the meanings of other elements related to them, will let us to draw the evolution of the acquatic imaginarium in Redonnnet's literary creation.
\end{abstract}

Keywords : minimalist writing ; waterly imaginarium ; river ; island ; ark.

Marie Redonnet (Paris, 1948) appartient à la génération d'écrivaines qui ont contribué au renouvellement du roman autour des années 80. Elle fait partie du contexte littéraire classé sous l'étiquette d' «écritures féminines», registre refuté par de nombreuses auteures revendiquant une écriture qui transcende les sexes. En tant que romancière Marie Redonnet débute avec la trilogie : Splendid hotel (1986), Forever Valley (1987) Rose Mélie Rose (1987), aux editions Minuit. En 2000 elle publie L'accord de paix, en 2005 Diego. Après dix ans de silence, elle revient sur la scène littéraire avec $L a$ femme au colt 45, un roman centré sur le destin d'une femme ayant choisi la liberté à la dictature.

L’univers romanesque de Marie Redonnet se caractérise par une écriture fluide et minimaliste qui cache des significations sous-entendues qui demandent un décryptage attentif de la part du lecteur. Cet univers allusif est chez Redonnet modulé par un système narratif qui ne permet pas au lecteur de se distraire avec la construction d'une intrigue complexe; au contraire, elle tient à ce que toute l'attention soit concentrée sur la découverte du caractère identitaire des personnages, de la valeur symbolique d' un geste anodin, d'une attitude nonchalante ou bien de la présence d'un objet banal, comme le pistolet dans La femme au colt 45. Dans ce roman Redonnet nous raconte d'une voix limpide, douée d'une immense force, l'existence d'une femme, Lora Sander, qui doit quitter son pays et subir le sort des réfugiés.

La structure narrative de La femme au colt 45 est atypique; le lecteur a l'impression de lire un long monologue -celui de Lora racontant son expérience vitale-, entrecoupé d' une sorte de voix off, nous explicitant les coordonnées spatiotemporelles. Cette structure nous fait penser à une pièce de théâtre où les déclamations à la première personne de l'actrice alternent avec les didascalies qui situent les lieux de l'action, le contexte. D'ailleurs, la dramaturgie occupe une place d'exception dans le récit puisque, en Azirie, Lora était comédienne au théâtre dirigé par son mari. Avant de quitter son pays, cet espace était une sorte de cocon douillet à l'intérieur duquel Lora se sentait protégée. La fermeture du théâtre et sa fuite sont perçus comme l'expulsion du paradis: « Le seul monde que je connaissais, c'était celui du Magic Théâtre. Je ne m'intéressais pas à ce qui se passait à l'extérieur. Et me voilà soudain lancée seule dans une vie inconnue et une histoire qui me dépasse » $(2016: 13)$.

L'incipit du roman situe notre protagoniste dans un ancrage spatio-temporel stratégique, assise au bord d'une falaise à pic, au moment de partir vers l'exil. À son départ, pour tout bagage, Lora ne porte qu'un pistolet, légué par son père: «Elle sort de son sac un vieux colt 45. Elle l'essuie précautionneusement avec un mouchoir, l'examine comme si elle voulait vérifier qu'il est en bon état » (2016 : 9). Ce cadeau joue le rôle de fil conducteur à caractère ambivalent. D’un côté, sa présence récurrente au long du roman, rattache Lora à son existence antérieure: "À quoi pensait-il en me le donnant? Il m’a appris à tirer quand j'étais petite. C'était comme un jeu qu'il partageait avec moi, le seul qu'il m’autorisait » (2016: 77); de l'autre, cet objet fétiche la protège et lui procure une identité : " Les chauffeurs des camions frigorifiques du camping, ils sont tous là dans le noir à me mater. Ils restent à distance et ne font pas un geste car ils savent que mon colt est chargé et que s'il y a en avait un qui s'avançait je n’hésiterais pas à tirer. Pour eux je suis la femme au colt 45. J’ai réussi à gagner leur respect » (2016: 53). 
À côté de ce leitmotiv, un autre un thème récurrent va accompagner le parcours de Lora tout au long du roman: il s'agit de l'élément aquatique. Cette présence obsédante de l'eau ne surprend pas le lecteur des romans de Marie Redonnet, puisque dans tous ses ouvrages nous découvrons l'image de l'eau avec une régularité surprenante. Diego commence et finit près de la crique d'Ambres; l'intrigue d'Accord de paix se déroule au port; dans Rose Melie Rose et Forever Valley la mer y est toujours présente. Le marécage est le responsable de tous les malheurs dans Splendid Hotel. Pourquoi Marie Redonnet choisit-elle d'écrire des œuvres dont l'histoire se déroule près de l'eau? Pourquoi dans ses récits nous trouvons toujours des bateaux, des ports, des pêcheurs ? Est-ce que leur présence n'est qu'une étrange coïncidence ? Chez Redonnet l'eau assume le rôle de personnage par excellence. Le marais, les cascades, le fleuve, la mer participent directement aux histories; leur présence regorge de significations qui exigent un décryptage de la part du lecteur.

Dans son étude L'eau et les rêves (1942 : 13) Bachelard signale : « l'eau est la métamorphose ontologique essentielle. L'être voué à l'eau est un être au vertige ». Cette caratéristique de l'élément liquide justifie la fascination que l'eau exerce sur l'imaginaire des écrivains. L'eau, en tant que composant des forces imaginantes est paticulièrement manifeste dans la femme au colt 45 , tous les événements importants de la vie de Lora ont comme témoin le milieu aquatique sous ses différentes manifestations.

Le fleuve est beaucoup plus large que là où je l'ai traversé. Il se mélange avec la mer. Au loin j'aperçois le phare. À chaque marée, les eaux du fleuve montent. Santaré est situé à la rencontre du fleuve et de la mer. Quand je suis assise au bord du fleuve, je sens l'odeur de la mer, une odeur de vase et d'algues qui me pique la gorge. J'entends au loin les sirènes des bateaux qui quittent le port et tout près de moi les cris des muettes qui remontent le fleuve. Je regarde le ciel presque toujours voilé à cause de la brume qui arrive de la mer (Redonnet, 2016 : 54).

Concernant l'élément aquatique, il est important de signaler que dans l'incipit du roman, Marie Redonnet ne fait pas référence à l'eau de façon explicite, elle est évoquée par le biais de l'allusion à la falaise à pic qui suggère indirectement la présence de la mer ou du fleuve. Assise au bord de la falaise, Lora réfléchit sur sa vie passée, pense à son avenir. Le vide qui s'ouvre sous ses pieds au bord de la falaise préfigure le gouffre baudelairien qui donne le vertige ${ }^{1}$.

J'ai besoin de me reposer avant de commencer la descente en direction du fleuve: Le sentier est escarpé et à pic. Moi qui ai le vertige, je vais être obligée de dominer ma peur. Il ne faut surtout pas que je glisse car je n'aurais rien pour me rattraper. Cette descente est bien plus périlleuse que la traversée de la forêt. Si je veux atteindre le fleuve, je ne peux pas l'éviter. C’est le seul passage (Redonnet, 2016 : 14).

Pour atteindre son pays d'accueil, il lui faudra quitter cette perspective d'en haut et retrouver le sentier qui descend jusqu'au fleuve qui sert de frontière naturelle entre les deux pays. Cette descente constitue la première étape de ce que nous pouvons considérer comme un véritable voyage initiatique, plein des péripéties et des embûches qui attendent Lora dans sa nouvelle existence; car, même si elle est déjà entrée dans la maturité, elle a toujours vécu sous la protection des hommes:

Je vais commencer une nouvelle vie toute seule hors de mon pays. Je ne m’y suis jamais sentie bien. C’est pour ça que je ne sortais pas du Magic Théâtre. Zuka était toujours à mes côtés comme mon ange gardien. C’est mon colt maintenant qui est mon ange gardien. [...] sous l'aile protectrice de Zuka, Giorgio ne pouvait pas s'affirmer, comme moi qui suis restée une petite fille même si je suis une femme de presque cinquante ans! Il est temps que je devienne enfin moi-même (Redonnet, 2016 : 27-28).

\footnotetext{
${ }^{1}$ D’après G. Durand (1992: 124) «le vertige est l'image inhibitrice de toute ascension, un blocage psychique et moral qui se traduit par des phénomènes psychophysiologiques violents. Le vertige est un rappel brutal de notre humaine et présente condition terrestre ».
} 
Chaque étape de son parcours est perçue par l'héroïne comme une nouvelle épreuve à surmonter pour conquérir sa liberté et acquérir une autre personnalité. La première épreuve est la plus difficile, puisque elle doit couper les liens avec l'espace sécurisé du Magic Théâtre, un microcosme virtuel, à l'abri des intempéries du monde extérieur, qui la maintenait attachée à une réalité trompeuse : « C’est maintenant que j’apprends à vivre [...] Je suis obligée de réformer mon caractère pour m'adapter à ma nouvelle situation. [...] À Santaré, contrairement à ce que croyait Zuca, le Magic Theâtre n'est pas une référence. Je ne peux pas compter sur mon CV » (2016 : 76).

Le passage vers ce nouvel état se fait à travers l'eau. Le fleuve, symbole de vie et de mort, de purification et de renouvellement, est un élément à dépasser pour atteindre l'autre monde. Difficile à franchir et gardé par des créatures cauchemardesques, le passage du fleuve est une épreuve incontournable pour atteindre la vie éternelle (Chevalier, Gheerbrant, 1982 : 449). Dans la femme au colt 45, le fleuve joue le rôle de frontière naturelle entre deux pays; mais pour Lora il symbolise, la naissance à un mode de vie différent. De ce point de vue, l'acte de se dépouiller des vêtements, devient une cérémonie du passage à un nouvel état : «Elle a enlevé son manteau, son bonnet et ses gants. Elle est debout sur un talus dominant le fleuve. [...] Dans la brume on entend le bruit des rames. On devine la barque du passeur en train de regagner l'autre rive au pied de la falaise » (2016:17).

La barque, symbole par excellence de la traversée, du passage qui relie deux rives, deux mondes différents, et qui en principe devrait conduire Lora au monde de la liberté, la mène, en réalité, au royaume des ombres et de la nuit. La figure du passeur, moderne représentation du personnage mythologique de Caron, est l'emblème de la mort: « Tout ce que la mort à de lourd, de lent, est aussi marqué par la figure de Caron. Les barques chargées d’âmes sont toujours sur le point de sombrer. [...] La barque de Caron sera ainsi un symbole qui restera attaché à l'indestructible malheur des hommes » (Bachelard, 1942 : 94). Dans la femme au colt 45 le passeur concentre en lui toute la méchanceté et la cruauté dont est capable l'être humain:

Quelle traversée désagréable! Quand je suis arrivée à bout de forces au bord du fleuve, le passeur m’attendait avec exaspération. [...] J'étais sa dernière passagère, il était pressé d'atteindre l'autre rive. Il y avait déjà douze femmes à bord. Toutes avaient subi des violences avant d'atteindre le fleuve. Elles en portaient les marques sur le corps et sur le visage. J’avais préparé une enveloppe avec la somme demandée pour la traversée du fleuve (Redonnet, 2016 : 18).

Lora va vite découvrir que le pays d'accueil ne s'avère pas être la terre promise, mais un lieu d'exploitation où les sanspapiers, se trouvent confrontés à la pauvreté et à la précarité du travail, avec la connivence impassible du fleuve.

\begin{abstract}
Un homme armé nous attendait sur l'autre rive du fleuve. Un pick-up était garé devant l'embarcadère. L'homme y fit monter les femmes. [...] il me demanda ma bague de fiançailles en échange de ma liberté. Grâce au diamant, j’ai échappé à l'usine où ces malheureuses ont été conduites pour y travailler comme des esclaves. La Santarie a beau être le pays de la liberté, les règles internationales du travail dans les usines situées le long du fleuve ne sont pas respectées (Redonnet, $2016: 19$ ).
\end{abstract}

L’image saisissante de Santaré, décrite comme « le point de rencontre des errances et des naufrages d'une humanité à la dérive » (2016 : 59), nous donne une idée de l’ampleur du drame des migrants : « À partir de maintenant je vis dans la clandestinité comme tous les étrangers sans papiers qui arrivent à Santaré par la mer encore plus que par le fleuve. Cette ville est comme un aimant qui les attire» (2016 : 59). Marie Redonnet dénonce cette situation en nous montrant le périple d'une femme qui se retrouve toute seule en terre inconnue, où elle devra prendre en main son destin, la solitude étant une condition nécessaire dans tout voyage initiatique : « Mes premiers pas au pays de la liberté. C’est un grand jour! Mais je n’ai personne avec qui fêter» (2016 : 21). Toute seule Lora devra développer des stratégies de survie, se réinventer chaque jour sans l'influence enrobante de son mari. Seule la compagnie de son colt 45, qui lui sert de blindage, «c'est mon colt maintenant qui est mon ange gardien » (2006 : 27) et la présence apaisante du fleuve lui tiendront compagnie. 
Même si je suis fatiguée, je retarde le moment d'aller dormir parce que j'aime être assise au bord du fleuve. C'est un moment de détente que j'attends avec impatience. Je pense à Zuca et à Giorgio comme si je les avais connus dans une autre vie dont le souvenir s'éloigne chaque jour un peu plus. Je m'accroche à ce souvenir pour garder la mémoire de ce que j'ai été. Sans cette mémoire, je serais perdue (Redonnet, 2016 : 54).

Ils sont les témoins muets de la transformation que Lora va subir; son parcours initiatique exigeant non seulement un changement de vêtements, mais également une transformation physique et l'oubli de l'héritage affectif de son existence antérieure, dont il est si difficile de se défaire : " Il y a des rides sur le front et à la commissure des lèvres. La peau commence à se flétrir. Le teint a perdu de son éclat. Le regard est grave et inquiet. Les traits du visage sont harmonieux, des sourcils épais, des lèvres charnues. L'expression est tendue. Cette femme que je ne reconnais pas, sans aucun fard, c'est moi » (2016: 14).

S'il existe un espace privilégié pour l'isolement et le recueillement, nécessaires pour se retrouver soi-même, cet endroit est bien une île. Par excellence symbole d'un centre spirituel (Chevalier, Gheerbrant, 1982: 519), l'île est essentiellement un lieu de ressourcement et de découverte de l'inconscient. Dans la symbolique de l'île, à l'idée de rupture avec le monde antérieur vient se joindre la notion d'abri, de paradis perdu, protégé, entouré d'eau pour aboutir à l'image du retour au giron maternel et de la re-naissance. La thématique de l'île a inspiré depuis l'Antiquité l'histoire littéraire. Dans des récits mythiques, comme l'Odyssée d'Homère, l'île s'associe naturellement à l'expérience initiatique qui attend Ulysse, avant de parvenir à Ithaque. L'île devient lieu d'aventures avec Robinson Crusoé de Defoe ou l'île au trésor de Stevenson. Ce n'est pas un hasard si Marie Redonnet situe juste en face de la ville de Santaré une île dont le nom est significatif: l'île aux oiseaux. Pour Lora habiter cette île représente une étape essentielle de son voyage personnel. Depuis son arrivée, Santaré a été pour elle une terre hostile où elle a vécu tous les malheurs de sa condition d'étrangère: « désormais je ne pourrais plus dire que je ne connais rien de la précarité ni des dangers que court une étrangère en situation irrégulière» (2016 : 1483). L'île aux oiseaux, au milieu du fleuve, qui est devenu son plus cher confident, prend la signification d'un lieu magique cachant un trésor : un bateau au nom emblématique: L'Arche de Noé. «Lora est assise au bord du fleuve. En face d'elle, un bateau est amarré à l'île aux oiseaux, au milieu du fleuve. Sur la coque, le nom du bateau : L’Arche de Noé. Lora a coupé ses cheveux très courts. Elle porte un vieux jean et un pull marin » (2016: 87).

Si la barque du passeur est un instrument d'exploitation des migrants qui cherchent une vie meilleure à étranger, l'Arche de Noé dirigée par Nina Pratz, une femme « habitée d'une flamme qui réchauffe et éclaire loin autour d'elle » (2016 : 88) se revêt de toutes les connotations positives d'un microcosme, à l'intérieur duquel les migrants trouvent le refuge et le soulagement qu'ils ne trouvent pas ailleurs.

Elle a enfin trouvé sa vocation et son projet, offrir un bateau, l'Arche de Noé, et une île aux jeunes naufragés de Santaré. Après avoir écouté son histoire et appris que j'allais bientôt être à la rue et sans travail, elle m'a proposé de participer à l'aventure de l'Arche de Noé. J’y apporterai mon expérience du Magic Théâtre en animant un atelier et en montant des spectacles. Elle rêve de créer un théâtre sur l'île. J'ai accepté sa proposition sans réfléchir et sans hésiter (Redonnet, 2016 : 88).

Lora a changé de perspective, à présent elle se trouve à l'île aux oiseaux, elle a devant elle la mer pour poursuivre son parcours initiatique, c'est de là qu'elle va prendre son essor et commencer à devenir elle-même.

Lora à l'extrémité de l'île, face à la mer.

J'aime regarder la mer depuis la pointe de l'île. L'estuaire est dangereux à cause des courants et des tourbillons. Les bateaux ne s’y risquent pas. Je fais le vide. Cette extrémité de l'île est le domaine des oiseaux. Leur vol et leur chant me grisent. Face à eux je ressens ma difficile et douloureuse condition humaine. J'ai fait un long voyage pour arriver jusqu'ici. Pour la première fois j'essaie d'exister par moi-même (Redonnet, 2016 : 93). 
C'est grâce au projet de l'Arche de Noé que Lora va commencer à voir la lumière au bout du tunnel ; dans l'espace sécurisé de l'île, à l'intérieur du bateau, elle est à l'abri de tous les dangers, entourée d'eau, liquide amniotique qui la protège du chaos de Santaré : «C’est une occasion inespérée et inattendue de refaire du théâtre. J'étais seule à Santaré et je vais me faire des amis dont je partagerai le projet. Je suis logée à bord du bateau. Dans ma cabine, j'ai l'impression de vivre entourée d'eau loin du chaos de Santaré » (2016 : 89).

La vie nouvelle qui se profile à l'horizon pour Lora acquiert toutes les caractéristiques d'une nouvelle étape dans cet itinéraire initiatique qu'elle poursuit. La libération à travers le théâtre est conçue comme une nouvelle cérémonie de renaissance à un nouvel état. L’image du néophyte balbutiant qui fait ses premiers pas est riche de signification. « Le travail théâtral tel que je le conçois serait à la fois une remémoration et une tentative de libération. Ce travail nous confronte chacun à nos résistances et à nos limites. Comme les jeunes de l'atelier, j'ai l'impression de balbutier et de manquer d'équilibre » (2016 : 94).

Mais le voyage n’est pas encore achevé. Pour se libérer et devenir elle-même, pour garder son équilibre et prendre son essor, Lora devra, -pour employer une image associée à la navigation-, jeter du lest, se débarrasser de tout ce qui la rattache à son passé, son colt 45 inclus. À mesure qu'elle grandit dans son nouvel état, le lien qui la rattache à cet objet évolue aussi. Marie Redonnet retrace l'évolution personnelle de Lora à travers le rapport qu'elle entretient avec son pistolet. Au début de sa vie clandestine, nous l'avons vu, le colt est pour elle un objet totémique qui la protège et lui donne une identité, même si elle est consciente que c'est aussi un objet dangereux : « Mon colt est autant un danger qu’une protection. Le marchant à qui je l'ai proposé n’a pas hésité à me l’acheter [...] C’est ainsi que je me suis séparée de mon colt. J'avais besoin de cet argent pour me louer une chambre. C’était ma priorité » (2016 : 65). Cependant, cette vente, n'étant pas un acte volontaire, est ressentie par Lora comme une trahison à la figure paternelle, qui la plonge dans le désespoir : « Je ressens un vide depuis que j’ai vendu mon colt. Rien ne peut le remplacer. C’est comme si j’avais perdu mon père une deuxième fois » (2016: 77).

Le lecteur est tout à fait conscient que Lora tient encore à cet objet, quoiqu'elle commence à entrevoir l'effet nocif de ce cadeau. «Il ne suffit pas que j’aie vendu mon colt. Même absent, comme la jambe d’un amputé, il continue de me faire du mal. Le cadeau de mon père ne pouvait être qu'empoisonné » (2016 : 90). Mais, grâce à sa forte détermination, petit à petit elle parviendra à se débarrasser du fardeau, de même qu'elle a su se passer de la protection de son patron et protecteur, Guido Rizi : «Les rendez-vous du jeudi soir, c’est fini ! À partir de maintenant je me passerai d’un protecteur comme je me passe de mon colt pour survivre à Santaré » (2016 : 84). Seulement, Lora ignore que pour rompre définitivement avec son passé, un dernier acte initiatique est nécessaire pour qu'elle puisse parachever son parcours. Le colt, comme un fantôme du passé, apparaît de nouveau en scène, véhiculant la catharsis qui délivrera définitivement notre protagoniste : Elle se précipite sur Niko pour le désarmer et s’emparer du colt. Au même instant Pipo se retourne et tire sur Nina qui
s’effondre. Elle tire sur Niko puis sur Pipo. Il s’effondrent à côté de Nina. [...]

Elle s’enfuit en courant, son colt à la main. Elle s’arrête au bout du ponton où sont amarrées les barques. [...]

Elle regarde fixement sont colt.

- Il devait me servir une dernière fois pour faire de moi une meurtrière. [...]

Elle jette son colt dans le fleuve.

- Maintenant que mon colt a accompli sa dernière mission, qu'il aille rouiller et pourrir au fond du fleuve. Et que j'en sois à jamais débarrassée (Redonnet, 2016 : 102).

Encore une fois le fleuve joue un rôle essentiel dans cette scène cathartique : l'effet corrosif de ses eaux, détruira définitivement le pistolet, débarrassant ainsi Lora des liens qui la maintenaient rattachée à son passé et à son influence maléfique. Une fois de plus, l'eau, associée maintenant au brouillard, sera l'élément purificateur qui accueille Lora après son meurtre : 
Elle monte dans une des barques amarrées au ponton.

Je dois aller à la police raconter ce qui vient de se passer. J'ai tiré en état de légitime défense pour sauver les jeunes de l'Arche de Noé. Je n'avais pas le choix. Maintenant je dois vivre avec le poids de mon geste. [...] Le brouillard est tombé d'un seul coup. La barque dérive. Le courant l'emporte vers la mer. Tout est-il déjà fini pour moi ? (Redonnet, $2016: 103)$

La question que se pose Lora, le lecteur se la pose aussi. À la fin du récit, il la retrouve à l’Aéroport. Qu'est-ce qui s'est passé depuis qu'elle devient une meurtrière jusqu'à ce moment-ci ? Comme Moïse, elle a été sauvée des eaux qui l'emportaient à la dérive. Après six mois à la prison des femmes, elle sera acquittée parce qu'elle avait tué en état de légitime défense pour sauver la vie des jeunes de l’Arche de Noé. En récompense de son acte héroïque elle obtiendra un permis de séjour pour vivre à Santaré. Dans cet intervalle, son mari est sorti de prison, après le renversement du dictateur de l’Azarie, et la réclame, mais sa décision est prise :

Je ne retournerai pas au Magic Théâtre jouer “la Reine de cœur” sous la direction de Zuca. Je ne suis plus celle que j'ai été. Je ne peux pas revenir en arrière comme si rien ne s’était passé. À Santaré j'ai commencé à me construire toute seule une nouvelle vie et une nouvelle personnalité [...]. C’est un choix personnel. Sans mon colt 45 maintenant qu'il rouille au fond du fleuve, je dois apprendre toute seule à devenir Lora Sander. Si je réussis j'aurai fait mes preuves.

Elle donne au chauffeur sa destination: l'embarcadère de l'île aux oiseaux (Redonnet, 2016 : 112).

Avec cette décision, Lora finit son parcours initiatique; tout ce qui la rattachait à son passé reste derrière elle. L'embarcadère de l'île aux oiseaux symbolise le point de départ d'une nouvelle vie pour une nouvelle Lora qui voit se profiler à l'horizon un avenir sans peur, sans attaches, rempli de nouveaux projets personnels.

\section{Références bibliographiques}

BACHelard, Gaston. (1942) L'eau et les rêves. Paris : José Corti

Chevalier, Jean et GheErbrant, Alain (1982). Dictionnaire des symboles. Paris : R. Laffont/Jupiter.

CotEA, Lidia (2005) Entre minimalisme et quête identitaire. L'imaginaire du corps dans l'œuvre de Marie Redonnet. Université de Bucarest : Edition Arvin.

Dambre, Marc et Blanckeman, Bruno (sous la direction) (2012). Romanciers minimalistes (1979-2003) Paris : Presses Sorbonne Nouvelle.

Demoulin, Laurent (1999). «Schoots (Fieke), “Passer en douce à la douane”. L'écriture minimaliste de Minuit : Deville, Échenoz, Redonnet et Toussaint », Textyles [En ligne] <http://textyles.revues.org/1276> [Consulté le 15 juin 2016].

Desanti, Jean-Toussaint (2004) La Peau des mots: Réflexions sur la question éthique. Conversations avec Dominique-Antoine Grisoni. Paris : Seuil.

DuRAND, Gilbert (1992). Les structures anthropologiques de l’imaginaire. Paris : Dunond.

Entretien avec Marie Redonnet. 2016. Marie Redonnet discute de La Femme au colt 45 avec Arno Bertina à l'invitation de la bibliothèque de Romainville et de l'Association des Bibliothèques en Seine Saint Denis dans le cadre des Matinées plateaux, préparatoires au festival Hors Limites. [En ligne], URL : <http://balises.bpi.fr/litterature/la-femme-au-colt-45-de-marieredonnet> [Consulté le 15 juin 2016].

Redonnet, Marie (1986a). Splendid hotel. Paris : Éditions de Minuit.

Redonnet, Marie (1986b). Forever Valley. Paris : Éditions de Minuit.

Redonnet, Marie (1987). Rose Mélie, Rose. Paris : Éditions de Minuit.

REDONNET, Marie (2016). La femme au colt 45. Paris : Le trypode.

SARrey-Strack, Colette (2002). Fictions contemporaines au féminin: Marie Darrieussecq, Marie Ndiaye, Marie Nimier, Marie Redonnet, Paris : Harmattan. 